\title{
Labview-Matlab integration for analyzing energy data of PV plants
}

\author{
S. Vergura, E. Natangelo \\ Dipartimento di elettrotecnica ed elettronica \\ Politecnico di Bari \\ Via E. Orabona 4, 70100 Bari (Italy) \\ Phone/Fax number:+0034 986 812685, e-mail: vergura@deemail.poliba.it, e.natangelo@yahoo.it
}

\begin{abstract}
The paper proposes the design of a Labview interface for acquiring, pre-processing, processing and visualizing data deriving from PhotoVoltaic (PV) plants. Some specific procedures for analyzing the energy performances of PV plants and implemented in Matlab environment have been integrated in the Labview interface. Its user-friendly approach allows the utilization of these complex routines to everybody. As voltage, current and energy of the DC side as well as of the AC side can give useful information about the operation of the PV plant (not only if failure is present but also if little misoperation is), the proposed interface allows also to compare the data of a specific date with other ones. The comparison with the data of the same date of a previous year allows to verify the trend of the performances, provided that similar environmental conditions exist. A real application is presented.
\end{abstract}

\section{Key words}

Photovoltaic, PV-plant, energy performances, statistical monitoring, energy data.

\section{Introduction}

The energy performances of PV plants are not easily evaluable because many variables affect their operation. The crucial problem is the strong dependence of the system response from factors, such as insolation intensity, ambient temperature, cell temperature, air velocity, humidity, cloudiness and pollution. Several models able to evaluate the effects of such uncertainties have been proposed and some standard benchmarks are usually used for evaluating energy performances of PV plants [1]. Unfortunately, these benchmarks show two drawbacks: a) they supply a rough information about the performance of the overall PV plant; b) they don't allow any assessment of the behaviour of the PV plant single parts. Some manufacturers of inverters and/or dataloggers realize softwares which give preliminary information about the operation of the PV plant. Usually, these softwares allow to evaluate only some basic statistics (mean, standard deviation, ecc.) and are protected by copyright; then it is not possible to interact with the data for utilizing advanced tools of statistics. Some specific reliability issues of PV plants are reported in [2] while [3] proposes a two-step procedure, implemented in Matlab environment to enhance the features of available softwares with advanced statistics. The former step consists in defining performance benchmarks and utilizes the population of the energy data (offline supervision). The latter one consists in verifying the well-operating of the PV plant and utilizes the sampled data of the energy data (real-time monitoring). Both the procedures, based on descriptive and inferential statistics, allow to detect and locate also little misoperations, which are not detected by evaluating standard benchmarks. Even if energy data of a whole year are not available, it is possible to extract information about the population applying other procedures described in [4]. The use of these procedures and the successive evaluation of the results are possible only for expert users, because they require specific skills. Moreover, Labview software is a user-friendly software with specific programming characteristics able to interact with Matlab environment. Then, the aim of this paper consists in presenting a Labview interface easy to be handled even if complex algorithms are implemented. The proposed interface is useful for the preliminary study of a PV plant operation as well as for statistical analysis of the energy data integrating the procedures previously described. For testing the proposed interface the same data of paper [3] have been used; then this paper shows only the characteristics of the interface while a comment about the results from a statistical point of view can be found in [3].

This paper is organized as follows: Section 2 presents Labview interface and output results for a specific case, Section 3 proposes the integration of the statistical procedures (programmed in Matlab) in Labview environment, while Section 4 reports the conclusions.

\section{Labview interface and output results}

Labview interface has been designed to visualize data of electric variables of PV plants. The data are usually stored in the datalogger at pre-fixed sampling time which can change during the day (10 or 15 minutes are standard values). Data almost always available are total daily energy, voltage on the DC side $\mathrm{Vdc}$, power on $\mathrm{AC}$ side Pac, and the total number of operation hours; Tab. I 
reports typical structure of available data in .xls format. The system under study for this specific case is installed on the roof top of a local authority owned multi-service company and is characterized by a rated power of 19,8 $\mathrm{kWp}$. It is a grid connected plant that injects the energy exceeding the typical consumptions of the company logistics into the distribution power network. The PV plant consists of 6 sub-arrays composed of two parallel connected strings, each of them having 11 photovoltaic $150 \mathrm{Wp}$ modules. The total power amount for a single array is of $3300 \mathrm{Wp}$.

Table I. Structure of data deriving from datalogger (inverter 1)

\begin{tabular}{|l|l|l|l|l|}
\hline & Vpv & Pac & E-Total & h-Total \\
\hline & V & W & kWh & H \\
\hline & Inv. 1 & Inv. 1 & Inv. 1 & Inv. 1 \\
\hline 01/07/2004 6:10:00 & 263 & 25 & 3394,473161 & 3234,409306 \\
\hline
\end{tabular}

The ActiveX technology integrated in Labview allows to take the data in .xls files and to store them in devoted structures (array and cluster).

After pre-processing and processing the data, some diagrams are available on the Labview interface:

- energy produced in the sampling time;

- cumulative energy produced;

- Vpv;

- Pac.

Figure 1 reports the energy produced in the sampling time from the beginning of the day; up the first diagram the date and the hour of last acquisition is indicated (red arrow).

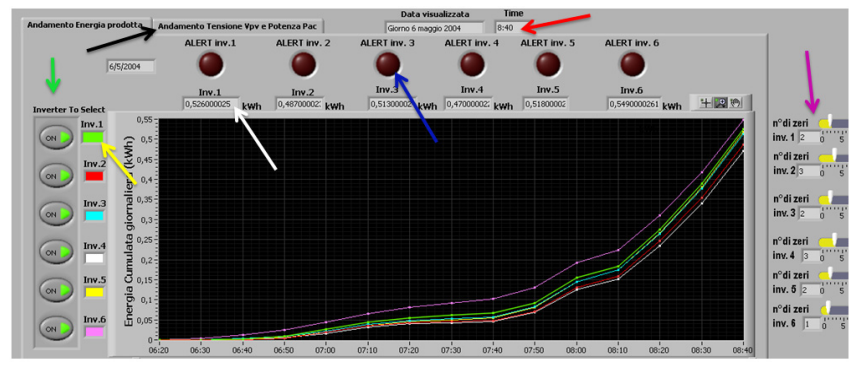

Figure 1: Front panel PV-plant monitoring program.

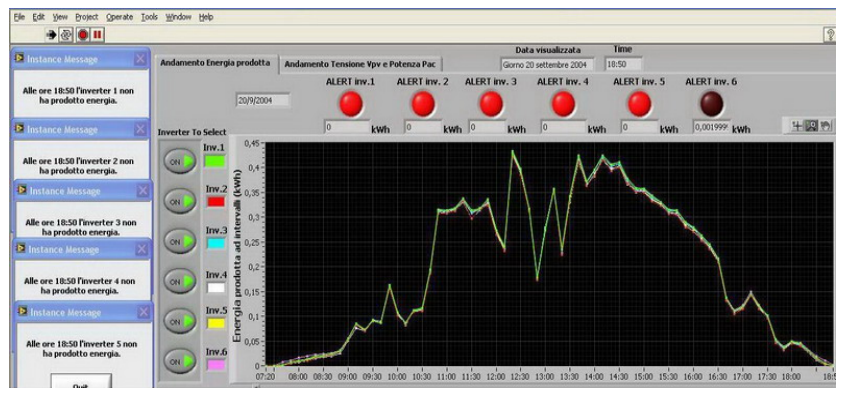

Figure 2: Example of 5 instance messages for inverters 1, 2, 3,

4,5 , which have not produced energy at 6:50 p.m.

Six curves corresponding to the six sub-arrays are reported for each diagram and they can be easily identified by stained squares on the left (yellow arrow).
Circles with green dots indicate that all the six sub-arrays are now visualized (green arrow); if necessary, a click on each button disables the visualization of the corresponding curve. Digital displays report the energy value of each sub-array (white arrow). Dark red circles are alert pointers which switch on (as in Figure 2) if there is no energy production (blue arrow). In this case, in the same an alert message is generated reporting the subarray which has not produced and the related time (Figure 2).

On the right of Figure 1 (fuchsia arrow) digital counters indicates the total number of zero-production for each sub-array and they are updated each time a new zeroproduction is present. Up on the left of Figure 1 there is the command to switch from energy diagrams to $\mathrm{Vpv}-\mathrm{Pac}$ diagrams (black arrow).
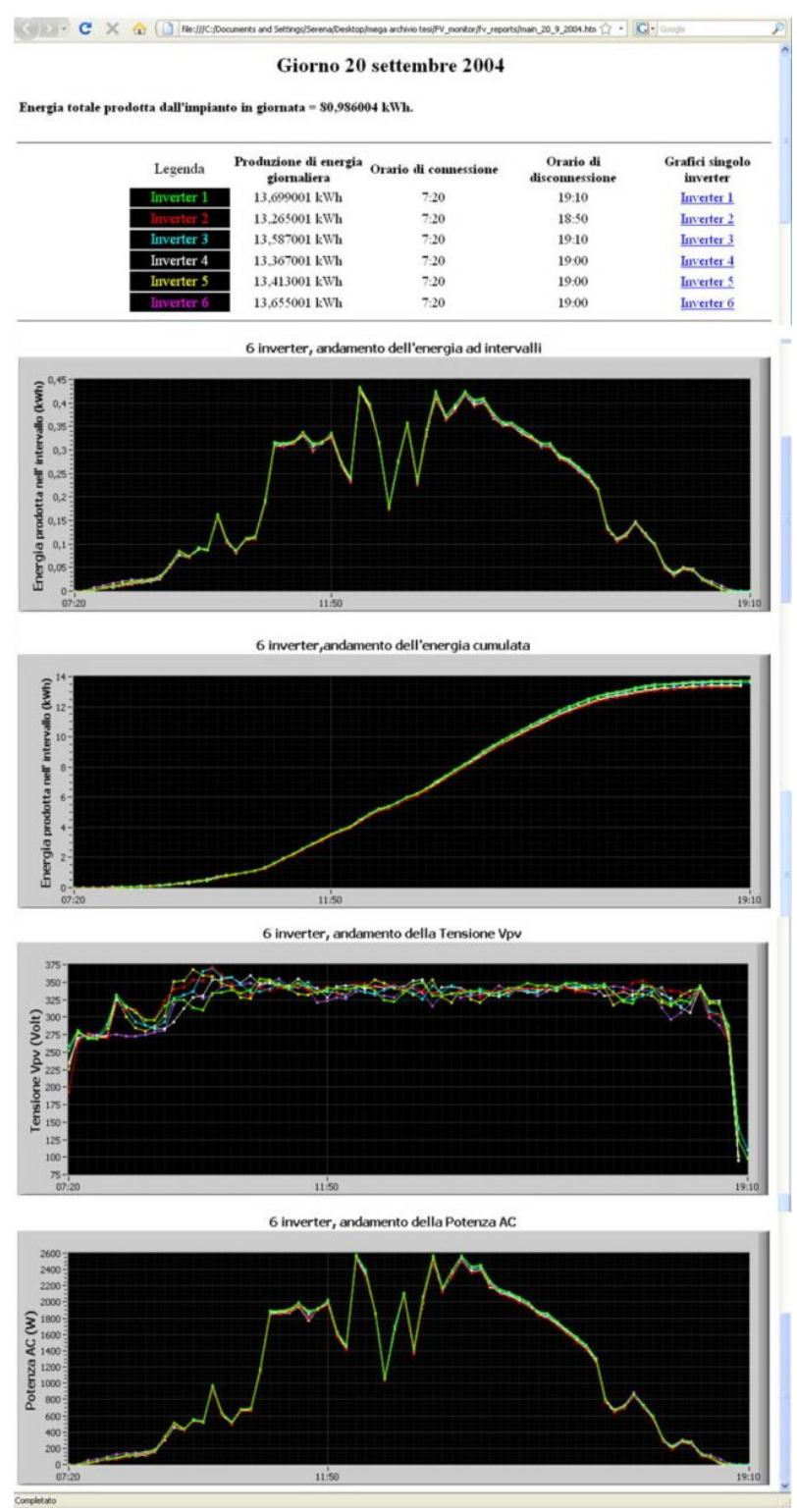

Figure 3: Main HTML report page for 20/09/2004.

At the end of each day, the software automatically generates a main HTML report page (Fig. 3) from which it is possible to choose the HTML report page for each inverter. Main report page is organized as follows:

- Column 1 - colour legend and inverter number; 
- Column 2 - daily produced energy;

- Column 3 - connection hour;

- Column 4 - disconnection hour;

- Column 5 - link to the data of each inverter.

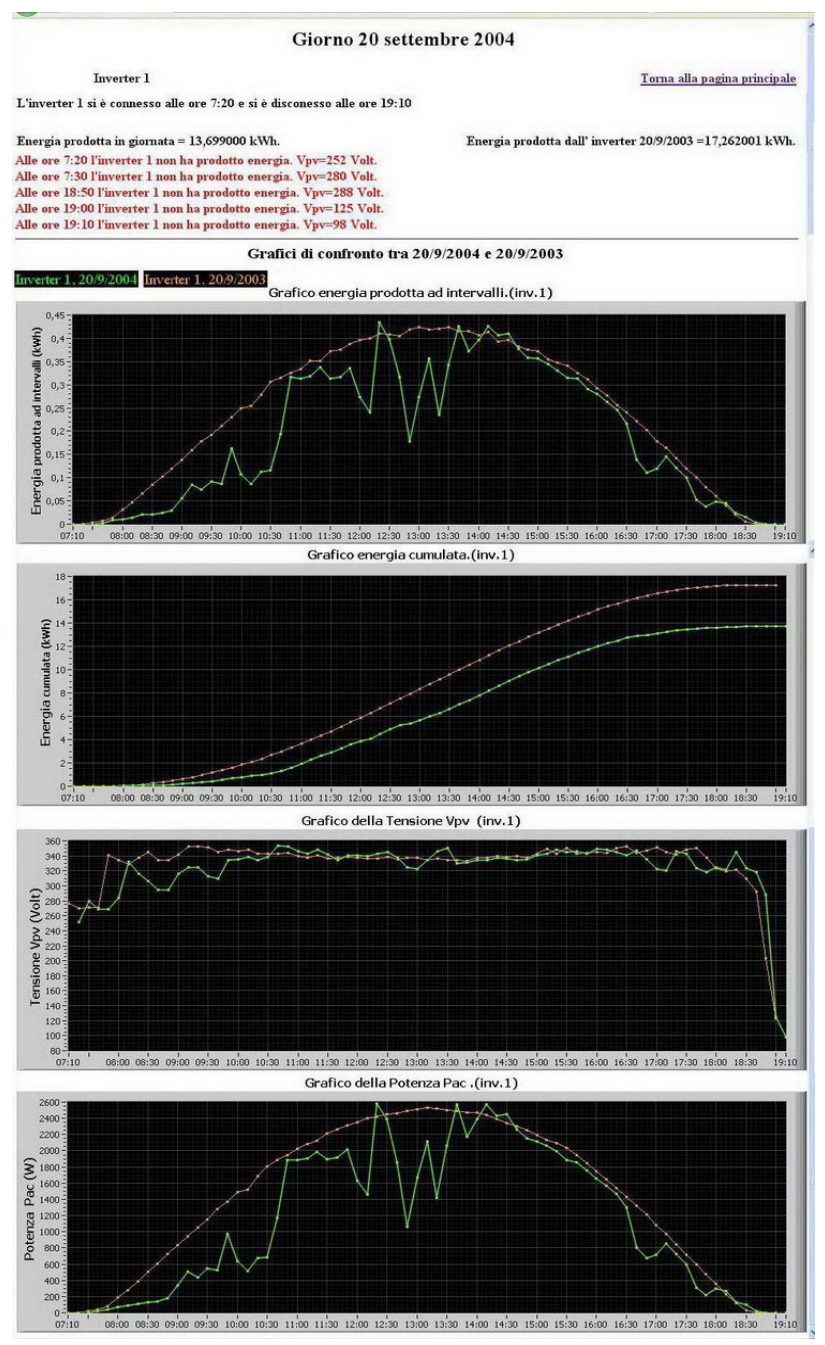

Figure 4: Report page of inverter 1 and diagrams of the present date and the date of the previous year.

Below the table the diagrams of partial produced energy, cumulative produced energy, Vpv and Pac are inserted.

Before generating HTML pages of a specific inverter it is possible to choose if a comparison with the electrical data of another date has to be realized. The comparison can regard only one inverter or all the inverters. A default choice is the same date of the previous year, but any date available can be chosen.

Figure 4 reports HTML page of inverter 1 for the date September 20th, 2004. The upper part reports both the connection and disconnection hours and the total daily produced energy (on the left) and the corresponding value of the previous year (on the right). Moreover, the instants of no-production with the related voltage value are reported. In Fig. 4 the diagrams of the daily energy for a specific date (September $20^{\text {th }}, 2004$ ) and the same date of the previous year are superimposed in order to verify the mismatches. Other parameters (radiance, ambient temperature, ecc.) are necessary for a detailed analysis if great mismatches are highlighted. The two curves in the 4 diagrams represent the same variable for the different date (in this case September $20^{\text {th }}, 2004$ and 2003).

\section{Labview-Matlab integration}

Procedures described in [3] can be applied on a yearlybased dataset as well as on a smaller dataset. The first approach allows to define the performance benchmarks for a PV plant in the real operation conditions. These benchmarks can be successively utilized for a comparison with new acquired data in order to evaluate if mismatches are present; the study in depth of the mismatches can reveal mis-operations before failure occurs. Those procedures are based on both descriptive and inferential statistics and have been implemented in Matlab environment. Using MATLAB script node of Labview, these procedures have been integrated in the user-friendly interface described in Sec. 2. The communication between the two software is based on ActiveX technology and is implemented in Windows environment.

Figure 5 shows the block diagram of the Labview application able to implement the statistical monitoring. The Flat Sequence function allows to define the right order of the instructions to be executed: the matrix of energy data is created in the first frame and successively it is placed in the MATLAB script node input, located in the second frame; then it executes the statistical analysis. The function Prompt For User Input is devoted to the interaction with the code; in fact, this last function allows to choice both the start and the final dates of the analysis. Successively this function allows to select the type of the temporal basis (daily or at intervals) to be used for the extraction of the data. If daily basis is selected, the daily total energy produced by each inverter will be calculated otherwise all energy data (usually acquired by datalogger for a sampling time of ten or fifteen minutes) will be used.

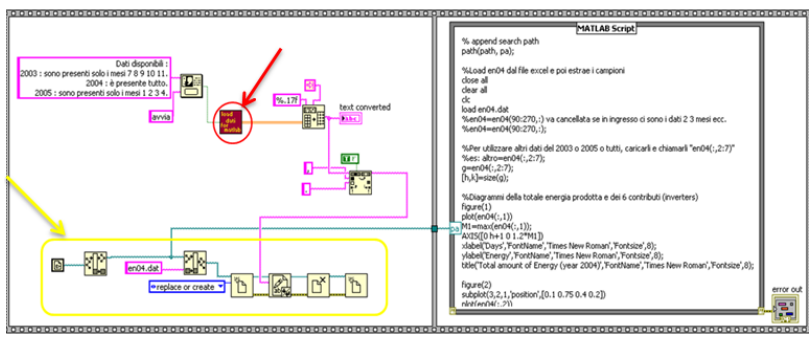

Figure 5: Block diagram of the statistical monitoring routine integrated in Labview

The red sub-routine of Fig. 5 creates the energy data matrix (each column is constituted by the data of each sub-array) which is used to execute the statistical analyses [3]-[4].

It implements the following functions:

1. generation of the addresses' array of the files in which the data are stored;

2. generation of the string-type matrix composed by six columns (one for every inverters). The elements of a row of the matrix are the 
spreadsheet strings associated with each subarray; it contains all monthly data stored in the datalogger and extracted from the .xls files. Each month belonging to the temporal window is selected;

3. Extraction of the energy data for every day of the temporal window selected. This operation depends on the chosen temporal basis. If daily basis is chosen, daily total energy produced by each sub-array of the PV plant is considered;

4. Generation of the data matrix whose the first column is constituted by the energy data produced by full PV plant and the following columns are constituted by the data of each subarray.

Finally, the energy data matrix is converted in a spreadsheet string with a specific format; then it is stored in the main directory to correctly interact with Labview interface. These instructions are highlighted in Fig. 5 by the yellow arrow, while Fig. 6 reports the details of the red sub-routine block-diagram.

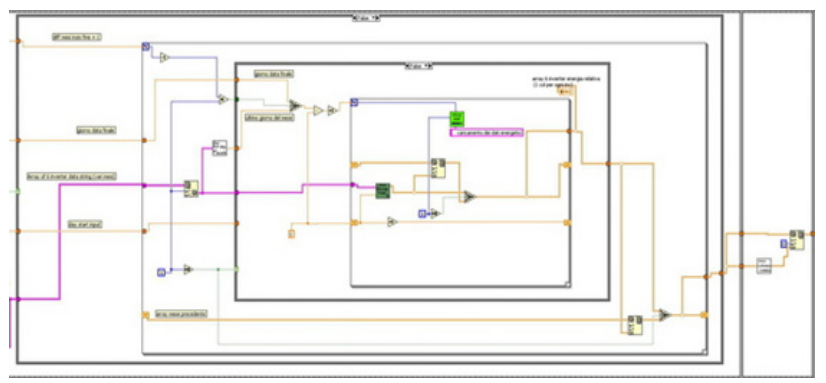

Figure 6: Block diagram of the red sub-VI of Fig. 5

The statistical procedure, based on descriptive and inferential statistics [3]-[4], utilizes the sampled data of the energy data and allow to detect and locate also little mis-operations, which are not detected by evaluating standard benchmarks.

\begin{tabular}{|c|c|c|c|c|c|c|}
\hline & \multicolumn{6}{|c|}{ Inverter number } \\
\hline & 1 & 2 & 3 & 4 & 5 & 6 \\
\hline Mean & 11.63 & 11.45 & 11.38 & 11.57 & 11.29 & 11.60 \\
\hline $\begin{array}{l}\text { Global } \\
\text { mean }\end{array}$ & \multicolumn{6}{|c|}{11.50} \\
\hline Spread \% & 1.13 & -0.44 & -1.04 & 0.61 & -1.83 & 1.04 \\
\hline Median & 12.53 & 12.13 & 12.32 & 12.21 & 11.94 & 12.24 \\
\hline $\begin{array}{l}\text { Global } \\
\text { mean }\end{array}$ & \multicolumn{6}{|c|}{12.22} \\
\hline Spread \% & 2.54 & -0.74 & 0.82 & -0.08 & -2.29 & 0.16 \\
\hline Variance & 39.31 & 39.41 & 38.60 & 40.05 & 38.52 & 41.00 \\
\hline $\begin{array}{c}\text { Ratio } \\
\text { highest/ } \\
\text { smallest }\end{array}$ & \multicolumn{6}{|c|}{1.064} \\
\hline
\end{tabular}

Figure 7: mean,median and variance of energy (in kWh) produced by each inverter and spread respect to the global values.

The statistical analysis is constituted by the following steps :

1. evaluation of the energy means, variances and medians of each inverter and comparison with the values of the entire plant (output results are reported in Fig. 7);

2. verification the homogeneity property of the energy (apply Levene's test);

3. application of the normality test to verify if the data belong to a normal distribution (output results are reported in Fig. 8);

4. evaluation of two indexes to assess how much the distribution of the dataset diverges from the normal distribution: skewness and kurtosis;

5. formulation as null hypothesis;

6. assignment of the significance level $\alpha$;

7. application of ANOVA test(if the conditions 1 . and 2. are satisfied) to highlight the presence of a mis-operation (output results are reported in Fig. 9)
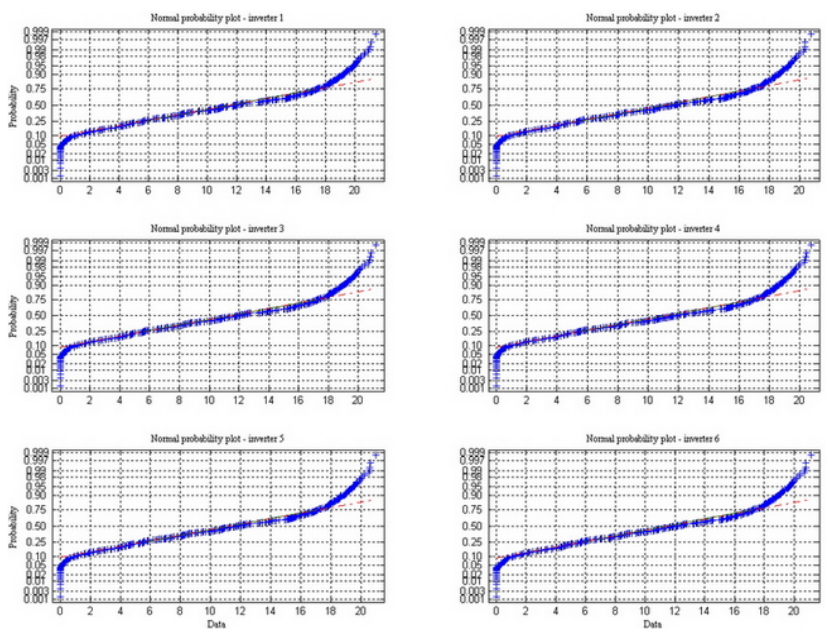

Figure 8: Normal probability plot for each one of the 6 inverters.

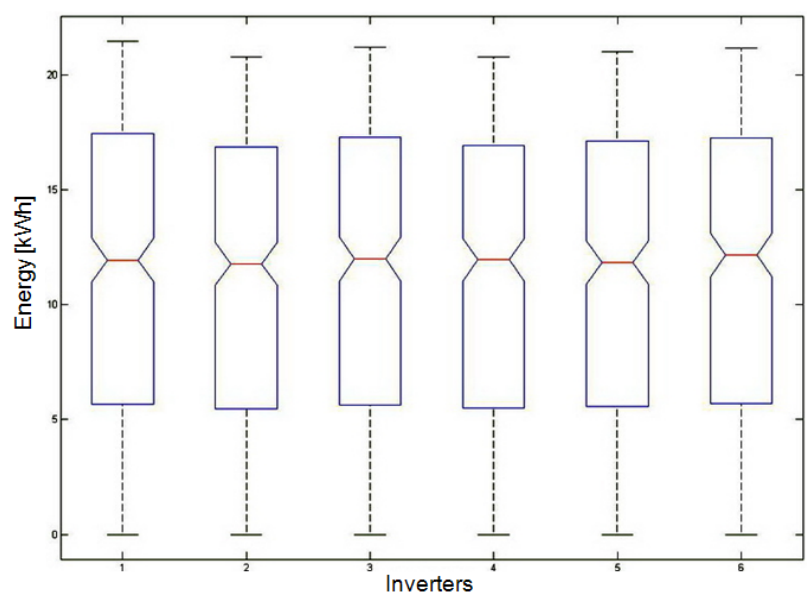

Figure 9: ANOVA test for the 6 inverters

\section{Conclusion}

User-friendly Labview interface to monitor energy performances of PV plants is presented. It allows to acquire available data, to visualize them, to inform the user in real-time whether a zero-production happens, to compare the diagrams related to another date for one inverter or for all the inverters, and to create HTML 
report pages for each inverter as well as for the whole PV plant.

Moreover, advanced procedures for monitoring the energy performances of PV plants and implemented in Matlab environment have been integrated in the operation of the proposed interface. These procedures can be applied when the data of a whole year are available as well as when they are not. In both cases descriptive and inferential statistics are used. The correct operation of the whole interface has been tested on the real data of PV plants.

\section{References}

[1] CEI-IEC International Standard 61724, "Photovoltaic system performance monitoring- Guidelines for measurement, data exchange and analysis", Ed. April 1998.

[2] G. Petrone, G. Spagnuolo, R. Teodorescu, M. Veerachary, M. Vitelli, "Reliability Issues in Photovoltaic Power Processing Systems", IEEE-Trans. On Industrial Electronics, Vol.55, issue 7, July 2008, pp. 2569-2580.

[3] S. Vergura, G. Acciani, V. Amoruso, G. Patrono, F. Vacca, "Descriptive and Inferential Statistics for Supervising and Monitoring the Operation of PV Plants",IEEE Trans on INDUSTRIAL Electronics, November 2009.

[4] S. Vergura, "Bootstrap Technique for Analyzing Energy Data from PV Plant”, IEEE-ICCEP 2009, Capri, Italy, pp. 268275 